\title{
Tuning Dimensionality by Nanowire Adsorption on Layered Materials
}

\author{
R. Adelung, J. Brandt, K. Rossnagel, O. Seifarth, L. Kipp, and M. Skibowski \\ Institut für Experimentelle und Angewandte Physik, Universität Kiel, D-24098 Kiel, Germany \\ C. Ramírez, T. Strasser, and W. Schattke \\ Institut für Theoretische und Astrophysik, Universität Kiel, D-24098 Kiel, Germany
}

(Received 21 March 2000)

\begin{abstract}
The dimensionality of electronic states determines a number of physical phenomena such as phase transitions, transport, or superconductivity. Employing scanning tunneling microscopy combined with angle-resolved photoemission spectroscopy we demonstrate how the dimensionality of electronic states can be continuously tuned from three to two dimensions. This is achieved by adsorption of nanowires on surfaces of layered crystals without changing the chemical composition of the material. Exemplary results for $\mathrm{Rb}$ nanowires on $\mathrm{TiTe}_{2}$ are discussed with the help of electronic structure calculations.
\end{abstract}

PACS numbers: 73.21.-b, 68.37.Ef

Engineering the electronic structure and, in particular, the fundamental band gap of semiconducting materials has emerged as a major tool for developing new electronic devices [1]. Band gap engineering is generally achieved by changing the chemical composition of, e.g., ternary semiconducting compounds like $\mathrm{Ga}_{x} \mathrm{Al}_{1-x}$ As. This way band gaps can be adjusted over the whole range of gaps from GaAs $(1.54 \mathrm{eV})$ to AlAs $(2.2 \mathrm{eV})$. However, the dimension of corresponding electronic states is generally not affected by tuning the band gap.

The dimensionality of the geometric and associated electronic structure of crystalline materials is responsible for a variety of interesting physical phenomena. For example, structural and/or electronic phase transitions associated with charge density waves and metal insulator transitions rely significantly on the dimension of corresponding electronic states [2]. In such materials Fermi vector nesting according to large parallel sheets of energy bands results in singularities of the dielectric susceptibility which is accompanied by periodic lattice distortions and charge density waves. Also it is generally believed that the quasi two dimensionality of electronic states in the vicinity of the Fermi level is responsible for the phenomenon of high $T_{C}$ superconductivity [3-5]. Thus, a stepless control of the dimension in the electronic structure without changing the chemical composition of the material would open up a wide field of electronic structure design and defined creation of associated physical properties such as, e.g., transport or superconductivity.

Tuning dimensionality, however, is a difficult task. Cutting a single layer out of a three-dimensional material is generally not suitable to reduce the dimension since such layers would require an additional stabilization. However, the basic building blocks of high $T_{C}$ materials are quasi-two-dimensional $\mathrm{Cu}-\mathrm{O}$ planes which are separated and stabilized by other more three-dimensional material. The degree of two dimensionality and consequently the superconducting properties can be controlled by the separation of the $\mathrm{Cu}-\mathrm{O}$ planes. Such additional stabilization can be avoided by taking stable layer structures as basic building blocks found, e.g., in the class of layered transition metal dichalcogenides. Their geometric structure is characterized by a three-dimensional stack of chalcogenmetal-chalcogen sandwich layers. The electronic structure of the material is three dimensional since comparable dispersion of electronic states perpendicular and parallel to the surface is observed [6]. It has been shown that the introduction of additional material between the layers can increase the layer separation (see, e.g., the work of Starnberg et al. [7] on $\mathrm{Cs}_{x} \mathrm{VSe}_{2}$ compounds). In consequence the overlap of electronic wave functions perpendicular to the layers is reduced. However, a freely tunable layer separation is not possible since the ionic radius of the added material principally determines the layer separation. In addition, the chemical composition of the materials is drastically changed thereby superposing the effects of changing dimensionality.

In this Letter we demonstrate how the dimensionality of electronic states can be effectively controlled by adding nanowires on the surfaces of layered transition metal dichalcogenides. $\mathrm{TiTe}_{2}$, a Fermi liquid reference compound, may serve as prototype material for designing the dimension of its well known electronic structure. $\mathrm{Rb}$ exposure to layered materials is employed to fabricate networks of metallic nanowires on the surface [8]. We show how these nanowires can be used to continuously tune the electronic decoupling of the surface layer from the bulk material. With increasing density of nanowires the dispersion of electronic states perpendicular to the sandwich layers can be flattened and finally destroyed to show pure two-dimensional behavior.

All results shown here have been obtained from clean and $\mathrm{Rb}$ exposed $\mathrm{TiTe}_{2}$ surfaces obtained by cleavage in ultrahigh vacuum. Rb was evaporated from SAES [9] getter sources employing a high flux. The resulting surfaces exhibit networks of $\mathrm{Rb}$ nanowires separating micrometer large flat areas in the surface layer. A typical STM image is shown in Fig. 1. The nanowires which are typically 5 


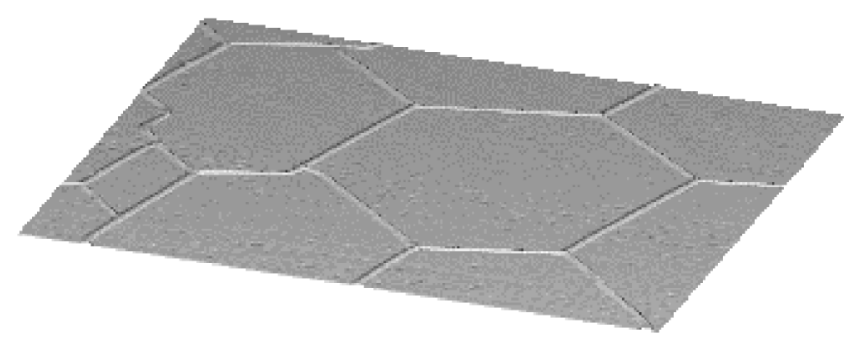

FIG. 1. STM empty state image (3D perspective view) of the alkali metal exposed $\mathrm{TiTe}_{2}$ surface. Height and width of $\mathrm{Rb}$ nanowires are typically $15 \mathrm{~nm}$ (image size $\sim 1500 \times 1000 \mathrm{~nm}^{2}$, bias $\left.=0.7 \mathrm{~V}, I_{t}=0.2 \mathrm{nA}\right)$.

to $100 \mathrm{~nm}$ thick revealing a width/height ratio of $\sim 1$ cut the surface layer into pieces. Note that the surface density of the $\mathrm{Rb}$ nanowires compared to the $\mathrm{TiTe}_{2}$ surface areas is only of the order of a few percent. Although the total amount of $\mathrm{Rb}$ contained in the wires would suffice to cover the surface with several monolayers, more than $95 \%$ of the substrate is uncovered. The morphology of the nanowire network such as wire thicknesses and mesh sizes can be tuned over a wide range. It should be noted here that all $\mathrm{Rb}$ atoms are contained in the wires. Neither adsorbed atoms nor atoms diffused into the bulk have been observed in STM or angle-resolved photoemission spectroscopy.

For comparison we show in Fig. 2(a) an STM image of a $\mathrm{Rb}$ exposed $\mathrm{WSe}_{2}$ surface close to the edge of the crystal where intercalation from the side is possible. The island structure of intercalated $\mathrm{Rb}$ is clearly visible. Such island structure has also been observed for $\mathrm{Na}$ intercalation
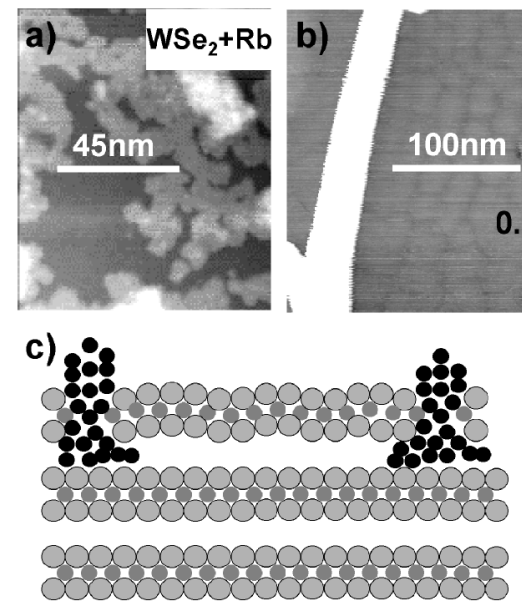
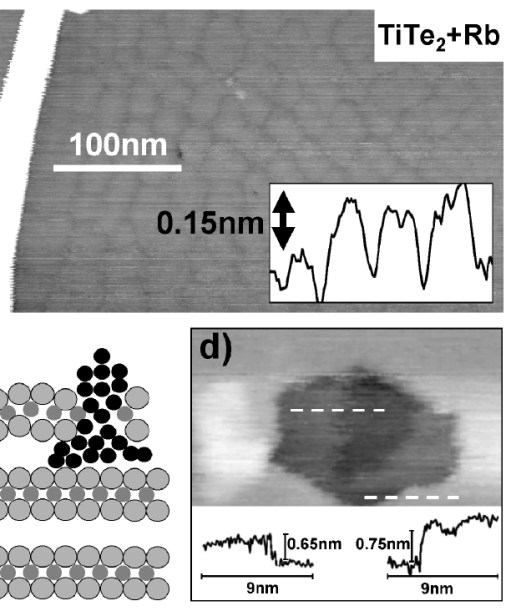

FIG. 2. (a) STM filled state image (bias $1 \mathrm{~V}, I_{t}=0.2 \mathrm{nA}$ ) of $\mathrm{Rb}$ exposed $\mathrm{WSe}_{2}$ surfaces close to the edge of the crystal showing intercalated $\mathrm{Rb}$ islands. (b) STM image exhibiting a $\mathrm{Rb}$ nanowire (white bar) which separates flat $\mathrm{TiTe}_{2}$ areas. The $\mathrm{TiTe}_{2}$ surface between the wires is slightly corrugated showing a wavelike pattern with varying amplitudes up to $0.2 \mathrm{~nm}$. The line scan shows the height profile along the scale bar. (c) Model of the topmost surface layer structure. $\mathrm{Rb}$ nanowires are depicted as coalesced black dots. Light and dark grey dots characterize chalcogen and metal atoms, respectively. (d) STM filled states image (bias $0.32 \mathrm{~V}, I_{t}=0.2 \mathrm{nA}$ ) of a hole in the $\mathrm{TiTe}_{2}$ surface. Line profiles reveal layer distances from 3rd to 2 nd (left) and 2nd to 1 st layers (right). in $\mathrm{VSe}_{2}$ [10]. This contrasts the images of $\mathrm{Rb}$ nanowires on $\mathrm{TiTe}_{2}$ [see Fig. 2(b)] where no buried islands of intercalated material can be found. Thus the STM shows no $\mathrm{Rb}$ atoms inside the meshes on top of the surface. Angle dependent $\mathrm{Rb}$ core level photoemission spectra, in addition, clearly indicate that no $\mathrm{Rb}$ atoms have diffused into the bulk [11]. This is further corroborated by calculations using density functional theory in the local density approximation for the exchange correlation functional and soft Troullier-Martins pseudopotentials [12] employing the FHImd98 code [13]. The diffusion barrier for Rb on the surface of $\mathrm{TiTe}_{2}$ is found to be $79 \mathrm{meV}$ [14]. This extremely low barrier provides the $\mathrm{Rb}$ atoms with a very high mobility at room temperature. $\mathrm{Rb}$ atom desorption off the wires is highly unlikely due to the relatively high activation barrier, yet the barrier for separating two $\mathrm{Rb}$ atoms of $507 \mathrm{meV}$ [15] is huge compared with the diffusion barrier of the single atoms.

The clean $\mathrm{TiTe}_{2}$ surface areas inside the meshes of the network show a wavelike structure induced by the surrounding wires. This is highlighted in the close-up of Fig. 2(b). A Rb nanowire separating flat $\mathrm{TiTe}_{2}$ areas is evident as white bar. The $\mathrm{TiTe}_{2}$ surface between the wires is slightly corrugated showing a wavelike pattern with varying amplitudes up to $0.2 \mathrm{~nm}$. The wavelike structures observed in STM are due to a modulation of the electron density which may be associated with a slight lattice distortion of the surface layer [16]. A model of the topmost surface layer structure is schematically depicted in Fig. 2(c) (side view). The surface layer between the wires (black dots) is corrugated. Subsequent layers are unaffected because of the weak van der Waals forces between the layers. It should be noted that the corrugation of the surface layer depends on the density of the nanowires. The higher the wire density is the larger the corrugation of the surface layer is. Although this corrugation looks like standing waves of a two-dimensional electron gas, it is mainly a pure geometric effect since the pattern does not change upon variation of the tip-sample bias in little steps between $+1.2 \mathrm{~V}$ to $-0.7 \mathrm{~V}$.

The corrugation of the surface layer is combined with a slight elevation of about $1 \AA$ compared to the bulk lattice constant which destroys the strong periodicity perpendicular to the layers. Figure 2(d) shows an STM image of a hole in the surface produced by a $5 \mathrm{~V}$ bias voltage peak. Corresponding line profiles across steps from the $3 \mathrm{rd}$ to 2nd (left) and 2nd to 1st layers (right) are depicted at the bottom. While the 3rd to 2 nd layer distance reveals the bulk lattice constant of $\operatorname{TiTe}_{2}(c=0.6498 \mathrm{~nm})$ the distance between the 2 nd and 1st layers is found to be larger by about $1 \AA$. Since the periodicity perpendicular to the layers is responsible for a three-dimensional dispersion of electronic states, an electronic decoupling of the surface layer is expected to lead to a vanishing dispersion of electronic states perpendicular to the layers.

Angle-resolved photoemission has emerged as an efficient tool to study the dispersion of electronic states in 
the Brillouin zone. We use the angle-resolved photoelectron spectrometer ASPHERE consisting of a hemispherical analyzer which is movable around two independent axes $[17,18]$. Applied with high resolution $(\Delta E=50 \mathrm{meV}$, $\Delta \vartheta=0.25^{\circ}$ ) and varying photon energies (supplied from the DORIS III storage ring at HASYLAB, DESY Hamburg) information on dispersion with respect to wave vector components perpendicular and parallel to the layers can be obtained. When exciting electrons from the Fermi level valuable information on the general shape of Fermi surfaces is accessible [19]. In Fig. 3 we show STM images and corresponding angle-resolved photoemission intensity maps scanning along wave vectors parallel to the surface by angular variation and perpendicular to the surface by photon energy variation [20] for clean and $\mathrm{Rb}$ nanowire network covered $\mathrm{TiTe}_{2}$ surfaces. Photoelectrons were excited from the Fermi level. Corresponding cuts through the bulk Brillouin zone schematically showing Fermi surfaces of 3D and 2D systems are plotted in the middle column. The top row shows data from the clean $\mathrm{TiTe}_{2}$ surface and the corresponding theoretical Fermi surface (middle). In particular, the Ti $3 d$ states coupling the layers over the van der Waals gap exhibit a pronounced dispersion with $k_{\perp}$. Upon adding nanowires to the surface and correspondingly tuning the level of electronic decoupling of the surface layer wave functions from the bulk the dimensionality can be engineered from 3D (top) over an intermediate level (middle) to pure 2D (bottom). It should be noted that although the dispersion perpendicular to the layers has vanished the dispersion parallel to the layers is mainly preserved. This is evident from the $2 \mathrm{D}$ photoemission intensity map of Fig. 3 (bottom) revealing different bands crossing the Fermi level (high intensities separated by low intensities [21,22]). Therefore, the observed electronic structure parallel to the layers resembles that of a crystalline layer. Because photoemission averages over a large (300 $\mu \mathrm{m} \times \mu \mathrm{m})$ area the sharpness of the intensity maps shows that the dimension "tuned in" by the nanowire coverage is found mainly on the whole sample. If there were domains of different dimensions they would produce a)
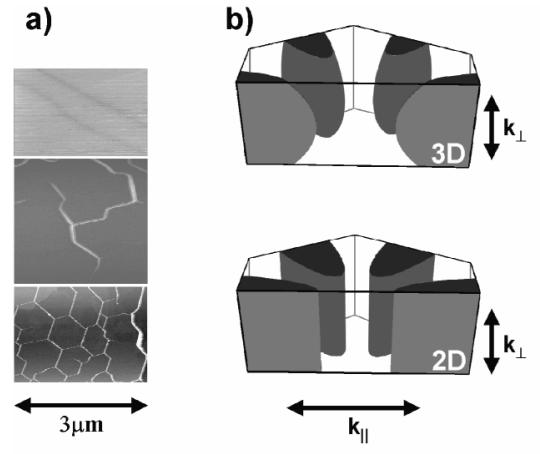
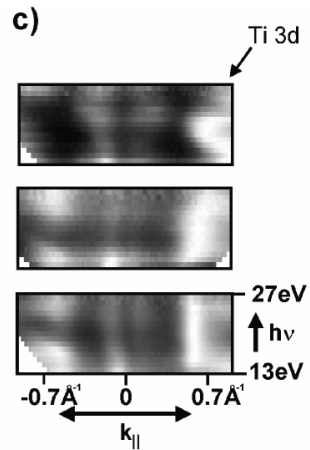

FIG. 3. (a) STM empty states images of clean (top) and Rb nanowire network covered $\mathrm{TiTe}_{2}$ surfaces (middle and bottom) (bias $=0.7 \mathrm{~V}, I_{t}=0.2 \mathrm{nA}$ ), (b) cuts through Brillioun zones schematically showing 3D and 2D Fermi surfaces, (c) corresponding angle-resolved photoemission intensity maps. a superposition of different dispersions perpendicular to the layers and consequently blur the images. It should be noted here that no $\mathrm{Rb}$ related features have been observed in photoemission energy distribution curves of this system as expected from the low coverage with $\mathrm{Rb}$ nanowires (few \%) compared to the large uncovered surface areas. It should be mentioned that identical behavior is observed for $\mathrm{Rb}$ nanowires on $\mathrm{TaS}_{2}$ [23].

A possible mechanism responsible for the observed loss of layer perpendicular dispersion could be found in the averaged elevation of the outermost Te-Ti-Te sandwich above its ideal position. This can explain the shift of the emission towards the $\Gamma$ point and the slight increase and sharpening of the central $p$ emissions, as shown below. The sharpness of the inner edge in comparison to the blurred outer edge of the $d$ emissions results from the sharp Fermi cutoff towards the $\Gamma$ point. To estimate the influence on the electronic structure empirical tight binding calculations (ETBM) have been performed for such a surface system by determining the layer, orbital, and wave vector resolved half-space Green function [24]. The electronic structure of the surface is characterized by the $k_{\|}$-resolved density of states (DOS) obtained from the Green function which offers a first estimate of the emission intensity.

Figure 4 shows the DOS for the first Te-Ti-Te sandwich. It is calculated at the Fermi energy and with $k_{\|}$along the $M^{\prime}-\Gamma-M$ direction. The thin line shows the result for the undisturbed crystal. Near the $\Gamma$ point, contributions from the Te $p$ orbitals and, near the $M$ and $M^{\prime}$ points,

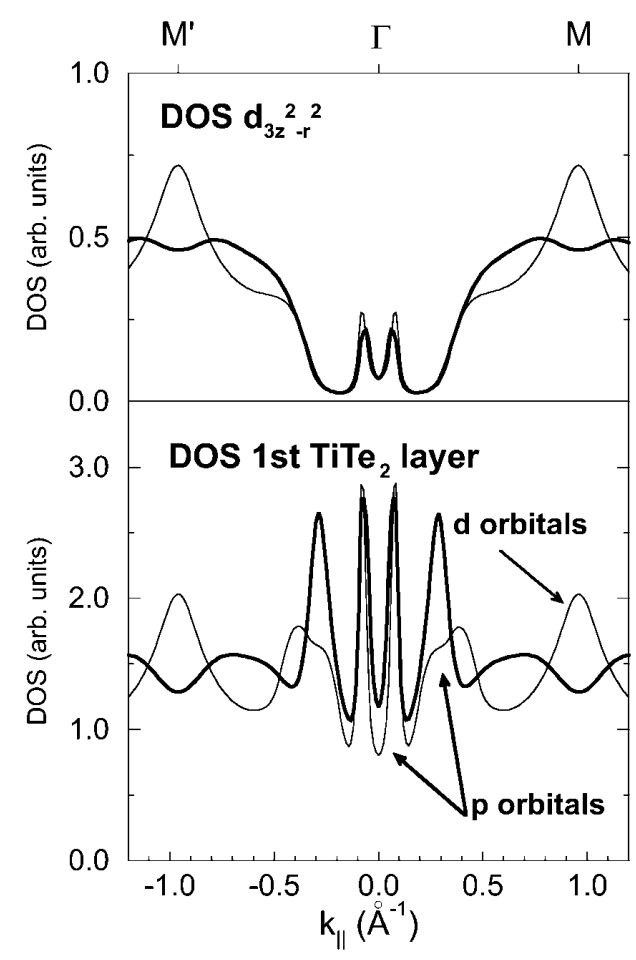

FIG. 4. Density of states of first Te-Ti-Te layer at Fermi energy. Thin line: undisturbed crystal, Thick line: first layer raised by $0.1 \mathrm{~nm}$ above ideal position and simultaneous increase of Fermi energy by $60 \mathrm{meV}$ (see text). 
contributions from the $\mathrm{Ti} d$ orbitals are visible. The thick line represents the DOS for the outermost Te-Ti-Te sandwich being raised by $0.1 \mathrm{~nm}$ with respect to the bulk crystal. Also, the Fermi energy has been locally increased by $0.06 \mathrm{eV}$ through distributing the additional electrons from the $\mathrm{Rb}$ atoms upon the first sandwich in a rigid bandlike model which, in fact, corresponds to a down bending of the bands at the surface and a constant Fermi energy throughout. The value of the shift is estimated from the part of the electrons distributed by the amount of $\mathrm{Rb}$ coverage and originate from the low work function of $\mathrm{Rb}$ compared with $\mathrm{TiTe}_{2}$. For simplicity a homogenous distribution is assumed though, in view of the observed STM pattern, the actual charge density of the Rb $s$ electrons might be modulated inside the meshes of the wire network according to the boundary conditions given by the remaining positive charge on the wires. In comparison with the undisturbed crystal the DOS peaks from the $d$ orbitals at about $\pm 1 \AA^{-1}$ are clearly shifted towards the $\Gamma$ point as it is found in experiment. The lowering of the amplitude and broadening which seems to contradict experiment with its reduced dispersion hides the theoretical behavior of the partial $d_{z^{2}}$ contribution to this peak. This, being much more important because it dominantly couples to the photocurrent via their matrix elements, displays a strong enhancement at the inner edge of this band in accordance with experiment. The DOS from exclusively $p_{x}$ orbitals at about $\pm 0.3 \AA^{-1}$ shows a significant narrowing and enhancement which can also be detected in the experimental data [note in Fig. 3(c) the transformation of the broad intensity next to the $k_{\|}=0$ line to a narrow structure closer to the $k_{\|}=0$ line]. Thus, the missing dispersion in $k_{\perp}$ and the sharpening and shift of the experimental structures are consistent with the calculated DOS and are to be expected to appear also in a one-step photoemission calculation yielding a possible explanation of the experimental results.

In conclusion, we have demonstrated that the adsorption of nanowires on flat surfaces of layered materials can be employed to reduce the dimension of electronic states. Depending on the density of nanowires on the surface the dimensionality can be continuously tuned from three to two dimensions. ETBM calculations of orbital and $k_{\|}$-resolved DOS reproduce this behavior when the topmost layer is slightly raised by about $1 \AA$. This opens up a procedure for designing physical properties such as phase transitions, charge density waves, or metal insulator transitions without changing the chemical composition of the material.

This research is supported by the BMBF, Germany (Projects No. 05 SE8 FKA and No. 05 SB8 FKB).

[1] S. M. Sze, Physics of Semiconductor Devices (Wiley, New York, 1981).

[2] K. Motizuki, Structural Phase Transitions in Layered Transition Metal Compounds (Reidel, Dordrecht, 1986).

[3] J. E. Hirsch and D. J. Scalapino, Phys. Rev. Lett. 56, 2732 (1986).
[4] D. M. Newns, H. R. Krishnamurthy, P.C. Pattnaik, C.C. Tsuei, and C. L. Kane, Phys. Rev. Lett. 69, 1264 (1992).

[5] V. Vescoli, L. Degiorgi, H. Berger, and L. Forro, Phys. Rev. Lett. 81, 453 (1998).

[6] O. Anderson, R. Manzke, and M. Skibowski, Phys. Rev. Lett. 55, 2188 (1985).

[7] H. I. Starnberg, H. E. Brauer, L. J. Holleboom, and H. P. Hughes, Phys. Rev. Lett. 70, 3111 (1993).

[8] R. Adelung, L. Kipp, J. Brandt, L. Tarcak, M. Traving, C. Kreis, and M. Skibowski, Appl. Phys. Lett. 74, 3053 (1999).

[9] Rb dispenser from SAES Getters, Lainate (Milano), Italy.

[10] I. Ekvall, H.E. Brauer, E. Wahlström, and H. Olin, Phys. Rev. B 59, 7751 (1999).

[11] Angle dependent photoemission spectra of Rb core levels changing the photoelectron escape depth clearly show increasing intensities when changing the emission angle from normal emission to higher polar angles (increased surface sensitivity).

[12] N. Troullier and J. L. Martins, Phys. Rev. B 43, 1993 (1991).

[13] M. Bockstedte, A. Kley, J. Neugebauer, and M. Scheffler, Comput. Phys. Commun. 107, 187 (1997).

[14] The calculations use the FHImd98 [13] code with TroullierMartins [12] pseudopotentials, to be published elsewhere.

[15] J. A. Kerr, in Chemical Rubber Company Handbook of Chemistry and Physics, edited by D. R. Lide (CRC Press, Boca Raton, FL, 1996), 77th ed.

[16] B.A. Parkinson, F.S. Ohuchi, K. Ueno, and A. Koma, Appl. Phys. Lett. 58, 472 (1991).

[17] K. Rossnagel, S. Harm, L. Kipp, and M. Skibowski, Nucl. Instrum. Methods Phys. Res. (to be published).

[18] L. Kipp and M. Skibowski, in Electron Spectroscopies Applied to Low-Dimensional Materials, edited by H.P. Hughes and H. I. Starnberg (Kluwer Academic Publishers, Dordrecht, 2000), Vol. 24.

[19] L. Kipp, K. Rossnagel, C. Solterbeck, T. Strasser, J. W. Schattke, and M. Skibowski, Phys. Rev. Lett. 83, 5551 (1999).

[20] Changing the photon energy generally changes $k_{\perp}$. For an absolute determination of $k_{\perp}$ the detailed dispersion of the final states has to be known which, however, is not important for the decision between strongly, weakly, and no dispersing bulk states. The maps in Fig. 3(c) are not expected to be symmetric for negative and positive $k_{\|}$because there is no mirror plane in $k$ space forcing this symmetry to be evident in photoemission spectra. In addition, the angle $\left(45^{\circ}\right)$ of light incidence gives rise to different matrix elements.

[21] The information of bands crossing $E_{f}$ is obtained from the detailed knowledge of the band structure from prior studies (see Ref. [20]) and many additionally recorded spectra of the nanowire covered samples.

[22] R. Claessen, R. O. Anderson, G.-H. Gweon, J. W. Allen, W. P. Ellis, C. Janowitz, C. G. Olson, Z. X. Shen, V. Eyert, M. Skibowski, K. Friemelt, E. Bucher, and S. Hüfner, Phys. Rev. B 54, 2453 (1996).

[23] R. Adelung, J. Brandt, L. Tarcak, L. Kipp, and M. Skibowski, Appl. Surf. Sci. 162, 666 (2000).

[24] J. Henk and W. Schattke, Comput. Phys. Commun. 77, 69 (1993). 